\title{
Chalcomoracin Prevents Vitreous-Induced Activation of AKT and Migration of Retinal Pigment Epithelial Cells
}

Haote Han 1,2, 3, 4, \#, Yanhui Yang 5, 3, 4\#, Bing Liu ${ }^{6,3,4}$, Jingkui Tian ${ }^{1,2}$, Wei Zhu ${ }^{1,2 *}$, Jiantao Wang ${ }^{7 *}$ and Hetian Lei ${ }^{7,3,4 *}$

${ }^{1}$ College of Biomedical Engineering \& Instrument Science, Zhejiang University, Hangzhou, 310027, P.R. China

${ }^{2}$ Zhejiang-Malaysia Joint Research Center for Traditional Medicine, Zhejiang University, Hangzhou, 310027, P.R. China

${ }^{3}$ Schepens Eye Research Institute of Massachusetts Eye and Ear, Boston, MA. USA

${ }^{4}$ Department of Ophthalmology, Harvard Medical School, Boston, MA, USA

${ }^{5}$ School of Basic Medical Sciences, Ningxia Medical University, Yinchuan, Ningxia, P.R.

China

${ }^{6}$ Guangzhou Women and Children's Medical Center, Guangzhou Medical University, 510005 Guangzhou, P.R. China

${ }^{7}$ Shenzhen Eye Hospital, Shenzhen Eye Institute, Jinan University, Shenzhen, Guangdong Province, P.R. China

*Correspondence: 18 Zetian Road, Shenzhen Eye Hospital, Futian District, Shenzhen, Guangdong Province, P.R. China. Email address: WZ:rutin@zju.edu.cn, JW:wangjiantao@126.com, HL: leihetian18@ hotmail.com 


\begin{abstract}
Retinal pigment epithelial (RPE) cells are the major cell type in the epi- or sub-retinal membranes in the pathogenesis of proliferative vitreoretinopathy (PVR), which is a blinding fibrotic eye disease and still short of effective medicine. The purpose of this study is to demonstrate if Chalocomoracin (CMR), a novel purified compound from fungus-infected mulberry leaves, is able to inhibit vitreous-induced signaling events and cellular responses intrinsic to PVR. Our studies have revealed that the CMR IC50 for ARPE-19 cells is $35.5 \mu \mathrm{M}$ at 72 hours, and that $5 \mu \mathrm{M}$ CMR inhibits vitreous-induced Akt activation and p53 suppression; in addition we have discovered that this chemical effectively blocks vitreous-stimulated proliferation, migration and contraction of ARPE19 cells, suggesting that CMR is a promising PVR prophylactic.
\end{abstract}

Key words: Chalocomoracin, Proliferative vitreoretinopathy, Vitreous, Akt, p53, Migration, Proliferation, Contraction

\title{
1. Introduction
}

Proliferative vitreoretinopathy (PVR) is a complication that can follow rhegmatogenous retinal detachments (RRD). There are approximately 55,000 individuals suffering from RRD in the United States annually [1, 2], and approximately 203,000 cases of open-globe injury worldwide every year [3]. PVR is still the major cause of failure after RRD surgery, and no relevant advances in clinical management have been made since the initial description [4]. In addition, so far there is no effective medicine for blocking the PVR formation [5-7]. Therefore, pharmacological approaches for PVR 
treatment are urgently demanding.

PVR was characterized by the growth of membranes on both surfaces of the detached retina and on the posterior hyaloids [1]. Retinal pigment epithelial cells (RPEs) are considered to be important and central players in the pathogenesis of PVR. When the retina is detached by contraction [8], retinal cells (e.g. RPEs) form the epi- or sub- retinal membranes undergoing a series of responses including proliferation and migration [9]. Hypothesis has been made that an eclectic range of chemical factors present within the vitreous fluid could be mitogenic to RPEs [10]. This hypothesis was supported by the observation showing that vitreous aspirated from patients with PVR was able to stimulate the migration of RPEs [11]. Moreover, vitreous fluid was shown to have an effect on cell proliferation which appeared to be mediated through TGF- $\beta$ [12]. Therefore, the vitreoustreated RPEs can be applied to in vitro studies of PVR.

Phosphoinositide 3 kinases (PI3Ks) play a critical role in experimental PVR [13] and regulate AKT, which is an oncogene product known as protein kinase B [14-16]. Activation of AKT can initiate multiple cellular processes such as cell survival, proliferation, growth, as well as migration [17, 18]. More recently, specific genetic variants located at p53 gene have been identified as significant risk factors for PVR development in patients $[19,20]$. Vitreous from experimental rabbits (RV) or vitreous from patients with PVR activate the signaling pathway of phosphoinsositide 3 kinase (PI3K)/Akt, which phosphorylates Mdm2, resulting in p53 degradation [21]. Prevention of Mdm2 interaction with p53 suppresses experimental PVR [22].

Chalocomoracin (CMR; MW: 648.69; structure, Fig. 1C) is a major secondary 
metabolite produced by fungus-infected mulberry leaves that protects the leaves by suppressing fungal germination [23]. Recently, CMR has been reported to have a broad spectrum of biological activities against rhinovirus, methicillin-resistant Staphylococcus aureus (MRSA) [24-26], and human cancer cell lines [27, 28], but there has not yet been any thorough investigation into its effects on PVR. Herein, we show that CMR inhibits vitreous-stimulated activation of Akt and abrogates vitreous-induced p53 suppression, proliferation, migration and contraction of ARPE-19, suggesting CMR is a promising medicine for prevention of PVR.

\section{Materials and methods:}

\subsection{Major reagents}

As determined by HPLC/UV-visible, $98.5 \%$ pure Chalocomoracin (CMR) was isolated by Jingkui Tian's lab, the Key Laboratory of Biomedical Engineering, Zhejiang University. The compounds were dissolved in pure DMSO and stored at $-20{ }^{\circ} \mathrm{C}$ prior to use. Primary antibodies against p-Akt, Akt and p53 were purchased from Cell Signaling Technology (Danvers, MA), and $\beta$-Actin was ordered from Santa Cruz Biotechnology (Santa Cruz, CA). Horseradish peroxidase-conjugated mouse anti-rabbit IgG, and goat anti-mouse IgG were ordered from Santa Cruz Biotechnology. Enhanced chemiluminescent substrate to detect horseradish peroxidase was purchased from Thermo Scientific (Waltham, MA).

ARPE-19 cells (American Type Culture Collection, Manassas, VA), was cultured in Dulbecco's modified Eagle's medium/nutrient mixture (DMEM/F-12, Invitrogen) 
supplemented with $10 \%$ fetal bovine serum (FBS). Cells were cultured in a humidified incubator at $37^{\circ} \mathrm{C}$ and $5 \% \mathrm{CO}_{2}$.

\subsection{Western blot}

This experiment was performed as described in previous reports [29]. Briefly, when cells were grown to $90 \%$ confluence in 24 -well plates, they were serum starved for $24 \mathrm{~h}$, and then treated with vitreous (0-24 h) from rabbits with PVR (RV, diluted 1:3 in DMEM/F12) or with CMR $(5 \mu \mathrm{M})$ for $8 \mathrm{~h}$. Proteins from treated cells were extracted in an extraction buffer and separated by $10 \%$ SDS-polyacrylamide gel electrophoresis. The proteins in the gel were then transferred to polyvinylidene difluoride membranes for western blotting analysis as described previously [30].

\subsection{Cell viability assay}

Cell viability was assessed by the 3-(4,5-dimethylthiazol-2-yl)-2,5-diphenyl-2Htetrazolium bromide (MTT, Solarbio, M8180). ARPE-19 cells were seeded into 96-well plates containing their respective medium supplemented with $10 \%$ FBS and incubated for $24 \mathrm{~h}$. The cells were then exposed to a range of concentrations of CMR $(0,5,10,20$ and $40 \mu \mathrm{M}$ ) dissolved in DMSO in 5\% FBS-supplemented medium for $24-72$ h. Following this, the medium was removed and replaced with $200 \mu \mathrm{L}$ of $0.5 \mathrm{mg} / \mathrm{ml}$ MTT in $10 \%$ FBS-containing medium, and the cells were incubated in the $5 \% \mathrm{CO}_{2}$ incubator at $37^{\circ} \mathrm{C}$ for $1 \mathrm{~h}$. Supernatants were removed from the wells, and the MTT dye was solubilized in $200 \mu \mathrm{L} /$ well DMSO (Sigma, 67-68-5). Absorbance was measured at $570 \mathrm{~nm}$ on a plate reader. Each condition was performed with 6 replicates and all assays were performed in 
triplicate, and the results of assays were presented as mean $\pm \mathrm{SD}$.

\subsection{Phase Contrast Imaging}

ARPE-19 cells were seeded into a 24-well plate containing their respective medium supplemented with $10 \%$ FBS and incubated for $24 \mathrm{~h}$. The cells were then exposed to a range of concentrations of $\operatorname{CMR}(0,5,10,20$ and $40 \mu \mathrm{M})$ dissolved in DMSO in 5\% FBS-supplemented medium for 24-72 h. The morphology of ARPE-19 was detected by EVOS Cell Imaging System.

\subsection{Cell proliferation assay}

This assay was performed as described in previous reports [29]. After ARPE-19 reached approximately $90 \%$ confluence, they were digested with $0.5 \%$ tripsin-EDTA, counted and plated into wells of 24 -well plates at a density of $3 \times 10^{4}$ cells/well in a full culture medium. After cells attached plates, they were treated with RV (1:3 dilution in DMEM/F12) in addition to CMR (5 $\mathrm{MM})$. On day 2 , the cells were trypsin digested and counted in a hemocytometer under a light microscope. At least three independent experiments were performed.

\subsection{Cell migration assay}

This assay was performed as described in previous reports [29]. Briefly, when ARPE19 was grown to confluence in $24-w e l l$ plates, autoclaved $200 \mu 1$ pipet tips were used to scratch the cells across the wells. The detached cells were aspirated out in PBS, and the rest cells were treated with RV (1:3 dilution in DMEM/F12) plus or minus CMR (5 $\mu \mathrm{M})$. 
The scratched areas were photographed to capture the initial width and photographed again $24 \mathrm{~h}$ later. The data were analyzed using Image J and Adobe Photoshop CS4 software as described previously [29]. At least three independent experiments were performed.

\subsection{Collagen contraction assay}

This assay was performed as described in previous reports [29]. In brief, when cells reached $90 \%$ confluence, they were re-suspended at a density of $1 \times 10^{6}$ in $1.5 \mathrm{mg} / \mathrm{ml}$ of neutralized PureCol type I bovine collagen solution (Advanced BioMatrix, San Diego, CA) ( $\mathrm{pH} \mathrm{7.2)} \mathrm{on} \mathrm{ice.} \mathrm{After} \mathrm{gently} \mathrm{mixed,} \mathrm{the} \mathrm{cells} \mathrm{in} \mathrm{the} \mathrm{collagen} \mathrm{gel} \mathrm{were} \mathrm{transferred}$ into wells of 24-well plates, which had been pre-incubated overnight with $5 \mathrm{mg} / \mathrm{ml}$ bovine serum albumin (BSA)/PBS. The mixture of cells and gel in the plates were incubated at $37{ }^{\circ} \mathrm{C}$ for $90 \mathrm{~min}$ for polymerization. Subsequently, $0.5 \mathrm{ml} \mathrm{DMEM} / \mathrm{F} 12$ or RV (1:3 dilution in DMEM/F12) with or without CMR $(5 \mu \mathrm{M})$ was added. On day 3 , the plates were photographed, and the gel diameter was measured. The gel area was calculated using a formula $3.14 \times \mathrm{r}^{2}$, where $\mathrm{r}$ is the radius of the gel.

\subsection{Statistics}

Data were analyzed as described preciously [29]. Data from at least three independent experiments were analyzed using ordinary one-way ANOVA followed by the Tukey honest significant difference (HSD) post hoc-test. p less than 0.05 was considered significantly difference.

\section{Results}




\subsection{Vitreous induces activation of AKT and reduction of p53 in ARPE-19}

Based on the previous reports, vitreous can promote the formation of PVR (Campochiaro et al., 1985). We used vitreous from PVR rabbits to establish a model in vitro. In terms of the activation of AKT, we found that RV (1:3 dilution in DMEM/F12) can significantly promote the phosphorylation of AKT after 15 minutes, and this process can be maintained for 8 hours, but the highest level achieves at $4 \mathrm{~h}$ (Fig. 1A and 1B). We mentioned earlier period during the formation of PVR, the expression of p53 was suppressed. Our results indicated that, in ARPE-19 treated with RV, obvious inhibition effect of p53 was observed after 30 minutes followed by the lowest level at $8 \mathrm{~h}$. Therefore, we chose $8 \mathrm{~h}$-treatment of RV in ARPE-19 for the following experiments.

\subsection{CMR prevents vitreous-induced activation of AKT and reduction of p53}

To find the potential effect of CMR for preventing PVR pathogenesis, we investigated the maximum-tolerated dose of CMR to ARPE-19. Thus, we treated cells with serially increasing concentrations: 5, 10, 20 and $40 \mu \mathrm{M}$. The results showed that $40 \mu \mathrm{M}$ CMR became obviously toxic to ARPE-19 cells indicated by morphology (e.g. cells shrinking and cell numbers) (Fig. 2A). In these cells, MTT assay (Fig. 2B) demonstrated that the $\mathrm{IC}_{50}$ of $\mathrm{CMR}$ was $35.5 \mu \mathrm{M}$ at $72 \mathrm{~h}$. However, $5 \mu \mathrm{M}$ CMR did not show obvious toxicity to ARPE-19 cells, which was assessed by morphology and MTT assay (Fig. 2).

To examine the impacts of CMR on the AKT/p53 pathway, we monitored phosphorylation of AKT at serine 473 as an indirect measure of activation of AKT. Based on previous study (Fig. 1 and Fig. 2), we treated ARPE-19 cells with CMR (5 $\mu \mathrm{M})$ 
in addition to RV for $8 \mathrm{~h}$. Western blot analysis of their lysates showed that CMR at 5 $\mu \mathrm{M}$ significantly blocked vitreous-induced activation of AKT (Fig. 3A and 3B) and suppressed vitreous-inhibited impact on p53 expression. These data demonstrate that CMR prevents the PVR pathogenesis focusing on inhibiting activation of AKT and suppression of P53, which is main molecular mechanism detected in PVR [17, 18, 20]

\subsection{CMR prevents vitreous-induced proliferation of ARPE-19}

The signaling events (PI3K/Akt signaling transduction) can trigger cellular responses such as cell proliferation. Thus, we examined if CMR could block vitreous-mediated cell proliferation, a characteristic of the pathogenesis of PVR [31]. As shown in Fig. 4, RV stimulated (1.6 \pm 0.2 fold) proliferation of ARPE-19, and CMR completely abrogated the RV-mediated cell proliferation.

\subsection{CMR blocks vitreous-induced migration of ARPE-19}

The signaling transduction from PI3K to Akt can stimulate cell motility and intracellular trafficking, while cell migration is an important event in the formation of PVR membranes. Therefore, we next investigated whether CMR could inhibit RVinduced cell migration. As shown in Fig. 5, RV stimulated migration of ARPE-19 in a wound-healing assay (Fig. 5A), and CMR significantly prevented this RV-induced cellular event through a dose-dependent way (Fig. 5B).

\subsection{CMR blocks vitreous-induced contraction of ARPE-19}


In the pathogenesis of PVR, another characteristic of PVR is the formation of PVR membranes whose contraction causes a retinal re-detachment [8]. So we attempted to apply a collagen gel contraction assay for mimicking this clinical event to discover drugs that can potentially prevent the pathogenesis of PVR. As predicted, vitreous promoted the contraction of ARPE-19 in a collagen contraction assay, and CMR completely blocked the contraction induced by the vitreous (Fig. 6A and 6B). Taken together, our findings indicate that CMR is a promising PVR prophylactic.

\section{Discussion}

Our results herein show that RV triggers the pathway of Akt/P53 in ARPE-19 cells, and that this pathway is critical for RV-stimulated cellular responses (e.g., proliferation, migration and contraction). More importantly, these experiments demonstrate that CMR inhibits vitreous-induced Akt activation and p53 suppression in ARPE-19 cells, and prevents vitreous-stimulated cell proliferation, migration and contraction of ARPE-19 cells in the pathogenesis of PVR. In this study, we used ARPE-19, which is a spontaneously arising RPE cell line derived from the normal eyes of a 19-year-old male donor, because these cells still express RPE specific markers including cellular retinaldehyde-binding protein (CRALBP) and RPE $65 \mathrm{kDa}$ protein (RPE65) [32, 33, 29].

The pathogenesis of PVR is divided into several stages: 1) migration of cells, mainly RPE and glial cells; 2) proliferation of the migrating cells; 3) membrane development; 4) contraction of the cellular membrane; 5) extracellular collagen production; and 6) creation of fixed folds in the retina [34]. Based on the idea that cellular proliferation is the main feature of PVR, many researchers have tried to solve this problem by inhibiting 
the proliferation of cells, but this problem remains unsolved. So, we can now focus on other mechanisms involved in PVR with the aim of developing a pharmacological approach for PVR treatment.

In our previous study, antioxidants were found to prevent intracellular signaling events that are essential for experimental PVR. For example, N-acetyl-cysteine (NAC) was shown to be capable of protecting rabbits from PVR [35]. Vitreous-driven activation of PDGFR $\alpha$ and cellular responses intrinsic to PVR (contraction of collagen gels and cell proliferation) are blocked by NAC [35], but the effect dose of NAC is too high (10 mM). Another report shows that idelalisib, a specific inhibitor of $\mathrm{PI} 3 \mathrm{~K} \delta$, specifically inhibits vitreous-induced Akt activation in RPE cells from epiretinal membranes from patients with PVR (RPEMs) without affecting Erk activation, as well as prevents vitreousstimulated cell proliferation, migration, and contraction of RPEMs [36]. Given that idelalisib is a specific inhibitor of $\mathrm{PI} 3 \mathrm{~K} \delta$, and the formation of PVR is a complicated process, the therapeutic effects of idelalisib on PVR are limited. Therefore, here we revealed for the first time that, as a natural compound, CMR has the advantage of effective, Multi-target and low toxicity, in terms of blocking vitreous-induced cellular responses contributes to PVR. CMR has been shown as a broad spectrum of biological activities, but there has not yet been any thorough investigation into the molecular mechanisms of CMR involved in different diseases except human reproductive system cancer. In our previous study, CMR induced paraptosis in combination with the mitophagy pathway in prostate and breast cancer cell lines without influencing the normal cells [28]. Then, we found that CMR could inhibit cell viability, proliferation, invation and migration of MDA-MB-231 cells (Fig. S1, Fig. S2 and Fig. S3). Therefore, 
we hypothesize that CMR can inhibit PVR which is closely related to proliferation and migration of RPEs. As predicted, in the current study, we found an inhibitory effect of CMR on Akt activation and p53 inactivation that was stimulated by vitreous in our study model (Fig. 7) and that that CMR repressed the vitreous-induced proliferation, migration and contraction of ARPE-19 cells.

PDGF-BB has been reported to be capble to induce the underlying pathways of Erk, p38, JNK activation in PVR [37], on the other hand, TGF $\beta$ induces epithelial mesenchymal transition (EMT) [38, 39] in RPEs. Thus, many signaling pathways are activated in PVR pathogenesis, of which especially Akt/MDM2/p53 among those signals is hyperactive in the virtreal environment $[29,40]$. Our data was clearly evident that CMR at $5 \mu \mathrm{M}$ completely blocked vitreous-induced activation of Akt, and p53 attenuation, however, whether it can suppress the activation of Erk, p38 or JNK induced by vitreous remains unknown. Furthermore, the impacts of CMR on vitreous-induced EMT also need further investigation. Overall, our work indicates CMR has therapeutic potential in prevention of vitreous-caused proliferative eye diseases such as PVR.

\section{Author contribution statement}

$\mathrm{HH}$ and YY performed most of the experiments, analyzed the results, and both made equal contributions to this work; BL performed some of the experiments; JT, WZ and JW conceived experiments and revised manuscript; HL conceived the experiments, analyzed the data and wrote the manuscript.

\section{Declaration of competing interest}


HH, JT and WZ have a patent with CMR, and all other authors declare that they have no conflicts of interest with the contents of this article.

\section{Acknowledgement}

This research was supported by China Scholarship Council (201806320148) to HH, the National Science and Technology Major Project of China (2019ZX09301004) and National Natural Science Foundation of China (81872973) to JT, by NIH grant R01EY012509 to H.L and Department Research to Prevent blindness to H.L. 


\section{References}

1. Hilton, G.; Machemer, R.; Michels, R.; Okun, E.; Schepens, C.; Schwartz, A. The classification of retinal detachment with proliferative vitreoretinopathy. Ophthalmology 1983, 90, 121-125.

2. Pennock, S.; Kim, D.; Mukai, S.; Kuhnle, M.; Chun, D.W.; Matsubara, J.; Cui, J.; Ma, P.; Maberley, D.; Samad, A.; Van Geest, R.J. Ranibizumab is a potential prophylaxis for proliferative vitreoretinopathy, a nonangiogenic blinding disease. Am. J. Pathol. 2013, 182, 1659-1670.

3. Morescalchi, F.; Duse, S.; Gambicorti, E.; Romano, M.R.; Costagliola, C.; Semeraro, F. Proliferative vitreoretinopathy after eye injuries: an overexpression of growth factors and cytokines leading to a retinal keloid. Mediators Inflammation 2013, 2013, 269787.

4. Pennock, S.; Haddock, L.J.; Eliott, D.; Mukai, S.; Kazlauskas, A. Is neutralizing vitreal growth factors a viable strategy to prevent proliferative vitreoretinopathy? Prog. Retinal Eye Res. 2014, 40, 16-34.

5. Assi, A.; Khoueir, Z.; Helou, C.; Fakhoury, H.; Cherfan, G. Intraocular application of Mitomycin $\mathrm{C}$ to prevent proliferative vitreoretinopathy in perforating and severe intraocular foreign body injuries. Eye (Lond) 2019, 33, 1261-1270.

6. Sohn, E.H.; Strohbehn, A.; Stryjewski, T.; Brodowska, K.; Flamme-Wiese, M.J.; Mullins,R.F.; Eliott, D. Posteriorly inserted vitreous base: preoperative characteristics, intraoperative findings, and outcomes after vitrectomy. Retina 2019.

7. Wang, H.F.; Ma, J.X.; Shang, Q.L.; An, J.B.; Chen, H.T.; Wang, C.X. Safety, pharmacokinetics, and prevention effect of intraocular crocetin in proliferative vitreoretinopathy. Biomed. Pharmacother. 2019, 109, 1211-1220.

8. He, H.; Kuriyan, A.E.; Su, C.W.; Mahabole, M.; Zhang, Y.; Zhu, Y.T.; Flynn, H.W.; Parel, J.M.; Tseng, S.C. Inhibition of proliferation and epithelial 
mesenchymal transition in retinal pigment epithelial cells by heavy chainhyaluronan/pentraxin 3. Sci. Rep. 2017, 7, 43736.

9. Schiff, L.; Boles, N.C.; Fernandes, M.; Nachmani, B.; Gentile, R.; Blenkinsop, T.A. P38 inhibition reverses TGFbeta1 and TNFalpha-induced contraction in a model of proliferative vitreoretinopathy. Commun. Biol. 2019, 2, 162.

10. Anderson, D.H.; Stern, W.H.; Fisher, S.K.; Erickson, P.A.; Borgula, G.A. The onset of pigment epithelial proliferation after retinal detachment. Invest. Ophthalmol. Visual Sci. 1981, 21, 10-16.

11. Campochiaro, P.A.; Jerdan, J.A.; Glaser, B.M.; Cardin, A.; Michels, R.G. Vitreous aspirates from patients with proliferative vitreoretinopathy stimulate retinal pigment epithelial cell migration. Arch. Ophthalmol. 1985, 103, 1403-1405.

12. Parapuram, S.K.; Chang, B.; Li, L.; Hartung, R.A.; Chalam, K.V.; Nair-Menon, J.U.; Hunt, D.M.; Hunt, R.C. Differential effects of TGF $\beta$ and vitreous on the transformation of retinal pigment epithelial cells. Invest. Ophthalmol. Visual Sci. 2009, 50, 5965-5974.

13. Lei, H.; Rheaume, M.A.; Velez, G.; Mukai, S.; Kazlauskas, A. Expression of PDGFR \{alpha\} is a determinant of the PVR potential of ARPE19 cells. Invest. Ophthalmol. Visual Sci. 2011, 52, 5016-5021.

14. Vanhaesebroeck, B.; Stephens, L.; Hawkins, P. PI3K signalling: the path to discovery and understanding. Nat. Rev. Mol. Cell Biol. 2012, 13, 195-203.

15. Songyang, Z.; Shoelson, S.E.; Chaudhuri, M.; Gish, G.; Pawson, T.; Haser, W.G.; King, F.; Roberts, T.; Ratnofsky, S.; Lechleider, R.J. SH2 domains recognize specific phosphopeptide sequences. Cell 1993, 72, 767-778.

16. Manning, B.D.; Toker, A. AKT/PKB signaling: navigating the network. Cell 2017, $169,381-405$

17. Vivanco, I.; Sawyers, C.L. The phosphatidylinositol 3-kinase AKT pathway in human cancer. Nat. Rev. Cancer 2002, 2, 489-501.

18. Luo, J.; Manning, B.D.; Cantley, L.C. Targeting the PI3K-AKT pathway in human cancer: rationale and promise. Cancer Cell 2003, 4, 257-262.

19. Rojas, J.; Fernandez, I.; Pastor, J.C.; Garcia-Gutierrez, M.T.; Sanabria, M.R.; Brion, M.; Coco, R.M.; Ruiz-Moreno, J.M.; Garcia-Arumi, J.; Elizalde, J.; Ruiz- 
Miguel, M. A strong genetic association between the tumor necrosis factor locus and proliferative vitreoretinopathy: the retina 4 project. Ophthalmology 2010, 117, 2417-2423.

20. Pastor-Idoate, S.; Rodriguez-Hernández, I.; Rojas, J.; Fernández, I.; GarcíaGutierrez, M.T.; Ruiz-Moreno, J.M.; Rocha-Sousa, A.; Ramkissoon, Y., Harsum, S.; MacLaren, R.E., Charteris, D. The p53 codon 72 polymorphism (rs1042522) is associated with proliferative vitreoretinopathy: the Retina 4 Project. Ophthalmology 2013, 120, 623-628.

21. Lei, H.; Velez, G.; Kazlauskas, A. Pathological signaling via platelet-derived growth factor receptor $\{$ alpha $\}$ involves chronic activation of Akt and suppression of p53. Mol. Cell. Biol. 2011a, 31, 1788-1799.

22. Lei, H.; Rheaume, M.A.; Cui, J.; Mukai, S.; Maberley, D.; Samad, A.; Matsubara, J.; Kazlauskas, A. A novel function of p53: a gatekeeper of retinal detachment. Am. J. Pathol. 2012, 181, 866-874.

23. Takasugi, M.; Nagao, S.; Masamune, T.; Shirata, A.; Takahashi, K. Chalcomoracin, a natural Diels-Alder adduct from diseased mulberry. Chem. Lett. 1980, 9, 1573-1576.

24. Nomura, T. Phenolic compounds of the mulberry tree and related plants. Prog. Chem. Org. Nat. Prod. 1988, 53, 87-201.

25. Fukai, T.; Kaitou, K.; Terada, S. Antimicrobial activity of 2-arylbenzofurans from Morus species against methicillin-resistant Staphylococcus aureus. Fitoterapia 2005, 76, 708-711.

26. Fukai, T.; Oku, Y.; Hano, Y.; Terada, S. Antimicrobial activities of hydrophobic 2 -arylbenzofurans and an isoflavone against vancomycin-resistant enterococci and methicillin-resistant Staphylococcus aureus. Planta Med. 2004, 70, 685-687.

27. Zhang, Q.J.; Tang, Y.B.; Chen, R.Y.; Yu, D.Q.; Three New Cytotoxic DielsAlder -Type Adducts from Morus australis. Chem. Biodiversity 2007, 4, 15331540.

28. Han, H.; Chou, C.C.; Li, R.; Liu, J.; Zhang, L.; Zhu, W.; Hu, J.; Yang, B.; Tian, J. Chalcomoracin is a potent anticancer agent acting through triggering Oxidative 
stress via a mitophagy-and paraptosis-dependent mechanism. Sci. Rep. 2018, 8, 114.

29. Chen, N.; Hu, Z.; Yang, Y.; Han, H.; Lei, H. Inactive Cas9 blocks vitreousinduced expression of Mdm2 and proliferation and survival of retinal pigment epithelial cells. Exp. Eye Res. 2019, 186,107716.

30. Lei, H.; Qian, C.X.; Lei, J.; Haddock, L.J.; Mukai, S.; Kazlauskas, A. RasGAP promotes autophagy and thereby suppresses platelet-derived growth factor receptormediated signaling events, cellular responses, and pathology. Mol. Cell. Biol. 2015, 35, 1673-1685.

31. Lei, H.; Rheaume, M.A.; Cui, J.; Mukai, S.; Maberley, D.; Samad, A.; Matsubara, J.; Kazlauskas, A. A novel function of p53: a gatekeeper of retinal detachment. Am. J. Pathol. 2012, 181, 866-874.

32. Dunn, K.C.; Aotaki-Keen, A.E.; Putkey, F.R.; Hjelmeland, L.M. ARPE-19, a humanretinal pigment epithelial cell line with differentiated properties. Exp. Eye Res. 1996, 62,155-169.

33. Samuel, W.; Jaworski, C.; Postnikova, O.A.; Kutty, R.K.; Duncan, T.; Tan, L.X.; Poliakov, E.; Lakkaraju, A.; Redmond, T.M. Appropriately differentiated ARPE19 cells regain phenotype and gene expression profiles similar to those of native RPE cells. Mol. Vision 2017, 23, 60-89.

34. Pastor, J.C.; Rojas, J.; Pastor-Idoate, S.; Di Lauro, S.; Gonzalez-Buendia, L.; Delgado-Tirado, S. Proliferative vitreoretinopathy: a new concept of disease pathogenesis and practical consequences. Prog. Retinal Eye Res. 2016, 51, 125155.

35. Lei, H.; Velez, G.; Cui, J.; Samad, A.; Maberley, D.; Matsubara, J.; Kazlauskas, A. $\mathrm{N}$-acetylcysteine suppresses retinal detachment in an experimental model of proliferative vitreoretinopathy. Am. J. Pathol. 2010,177, 132-140.

36. Xin, T.; Han, H.; Wu, W.; Huang, X.; Cui, J.; Matsubara, J.A.; Song, J.; Wang, F.; Colyer, M.; Lei, H. Idelalisib inhibits vitreous-induced Akt activation and proliferation of retinal pigment epithelial cells from epiretinal membranes. Exp. Eye Res. 2020, 190, 107884. 
37. Zhang, H.; Shang, Q.; An, J.; Wang, C.; Ma, J. Crocetin inhibits PDGF-BBinduced proliferation and migration of retinal pigment epithelial cells. Eur. J. Pharmacol. 2019, 842, 329-337.

38. Takahashi, E.; Fukushima, A.; Haga, A.; Inomata, Y.; Ito, Y.; Fukushima, M.; Tanihara, H. Effects of mechanical stress and vitreous samples in retinal pigment epithelial cells. Biochem. Biophys. Res. Commun. 2016, 470, 569-574.

39. Heffer, A.M.; Proaño, J.; Roztocil, E.; Phipps, R.P.; Feldon, S.E.; Huxlin, K.R.; Sime, P.J.; Libby, R.T.; Woeller, C.F.; Kuriyan, A.E. The polyether ionophore salinomycin targets multiple cellular pathways to block proliferative vitreoretinopathy pathology. PloS one 2019, 14.

40. Han, H.; Chen, N.; Huang, X.; Liu, B., Tian, J.; Lei, H. Phosphoinositide 3-kinase $\delta$ inactivation prevents vitreous-induced activation of AKT/MDM2/p53 and migration of retinal pigment epithelial cells. J. Biol. Chem. 2019, 294, 1540815417. 
A

ARPE19

Fig. 1

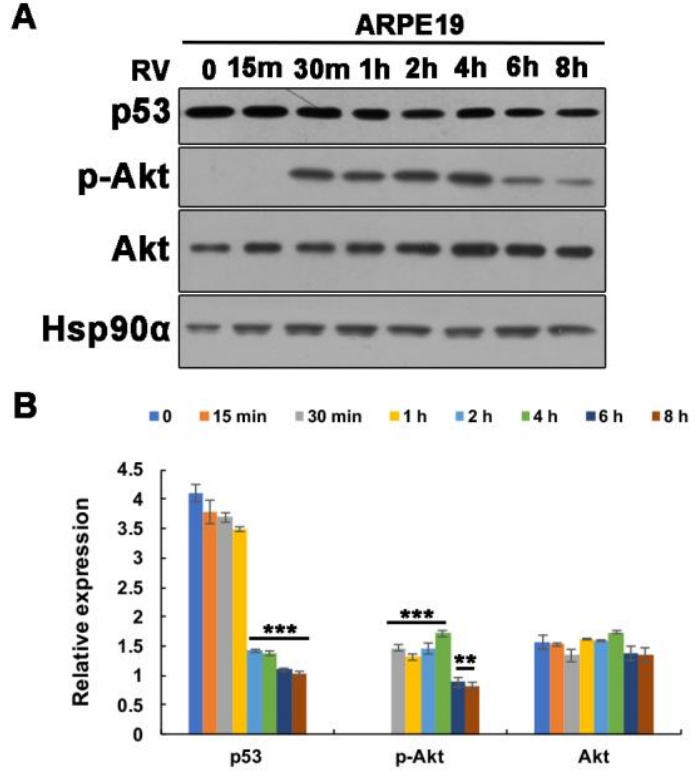

Fig 1. Vitreous induces activation of AKT and reduction of p53 in ARPE-19

(A) Serum-starved ARPE-19 cells were treated with vitreous for $15 \mathrm{~min}, 2 \mathrm{~h}, 4 \mathrm{~h}, 6 \mathrm{~h}, 8 \mathrm{~h}$, 10 h, 12 h and 24 h. RV: experimental-rabbit PVR vitreous diluted 1:3 in DMEM/F12. Their lysates were subjected to western blotting analysis using indicated antibodies. This is representative of at least three independent experiments.

(B) The intensity of the p-Akt, Akt and p53 bands. The bar graphs are mean \pm SD of three independent experiments. The data was analyzed using one-way ANOVA followed by the Tukey HSD post hoc-test. **p $<0.01, * * * p<0.001$. 
Fig. 2
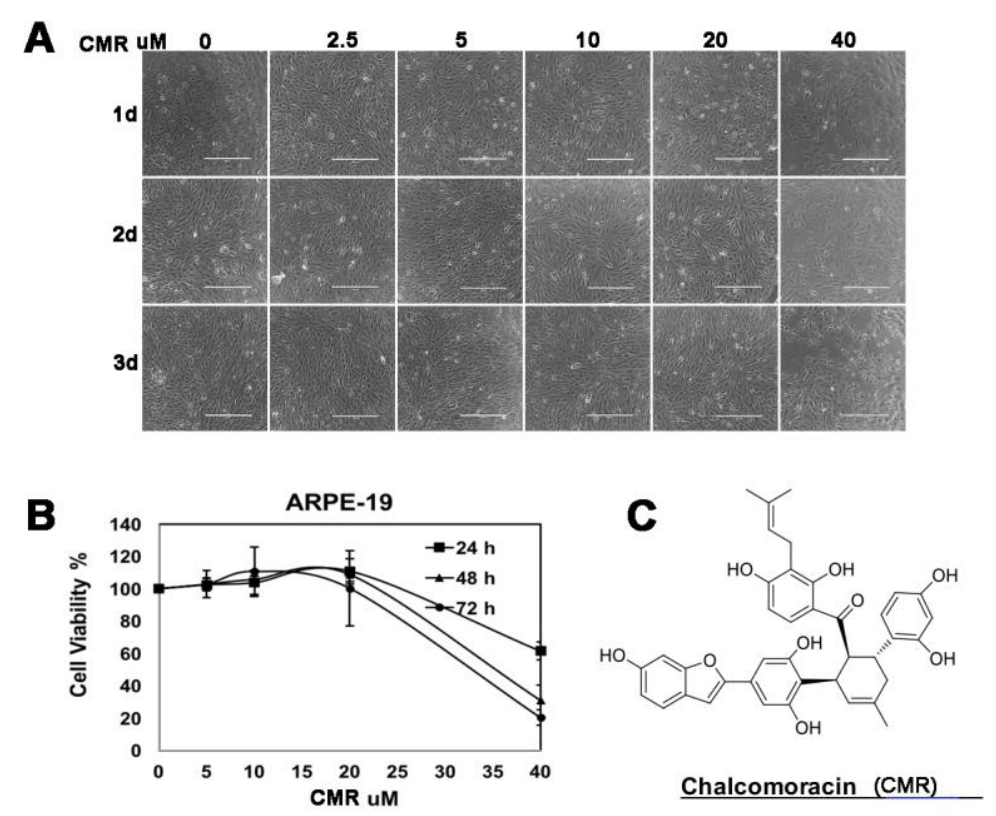

Fig 2. Evaluation of CMR toxicity to ARPE-19 cells

(A) Confluent ARPE-19 in a 24 -well plate were treated with a serially increasing concentration of CMR (V, 2.5, 5, 10, 20 and $40 \mu \mathrm{M})$ in 5\% FBS DMEM/F12. The images shown were taken of ARPE-19 treated for $24 \mathrm{~h}, 48 \mathrm{~h}$ and $72 \mathrm{~h}$. V: vehicle $0.1 \%$ DMSO. Scale bar: $200 \mu \mathrm{m}$. This is representative of three independent experiments.

(B) Dose-dependent inhibition on the viability of ARPE-19 was detected by MTT assay, following treatment for 24-72 hours in 5\% FBS-supplemented medium. Points: mean; bar: SD. Data are expressed as percentages of viable cells (treated vs. control). All assays were performed in triplicate. (C) CMR structure. 


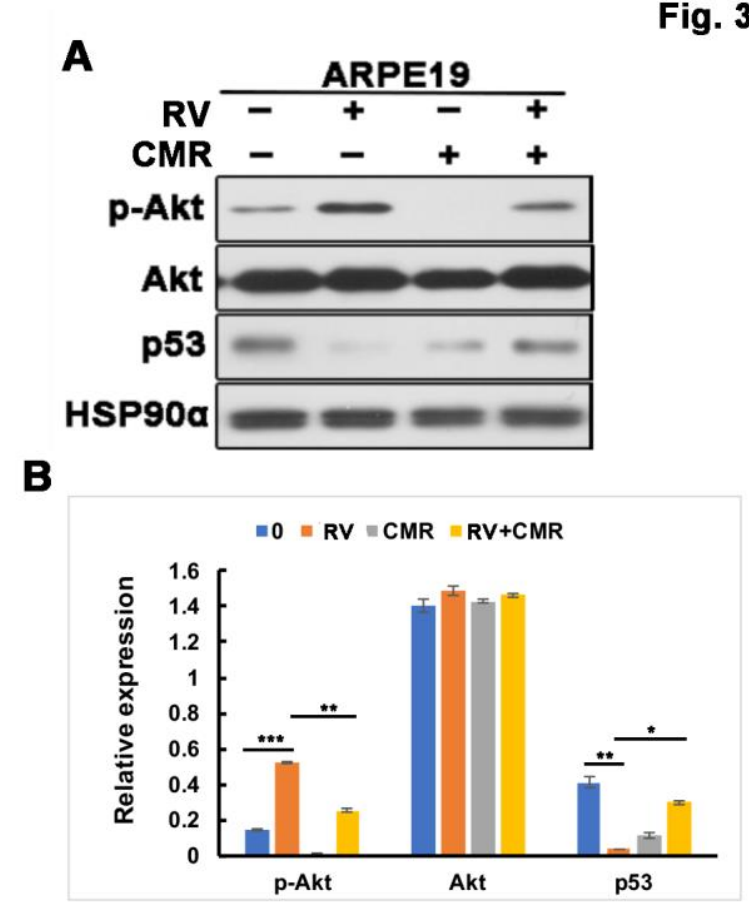

Fig 3. CMR prevents vitreous-induced activation of AKT and reduction of p53

(A) Serum-starved ARPE-19 cells were treated with vitreous in addition to CMR $(5 \mu \mathrm{M})$ for 8 h. RV: experimental-rabbit PVR vitreous diluted 1:3 in DMEM/F12. Their lysates were subjected to western blotting analysis using indicated antibodies. This is representative of at least three independent experiments.

(B) The intensity of the p-Akt, Akt and p53 bands. The bar graphs are mean \pm SD of three independent experiments. The data was analyzed using one-way ANOVA followed by the Tukey HSD post hoc-test. *p $<0.05$, **p $<0.01$, ***p $<0.001$. 
Fig. 4

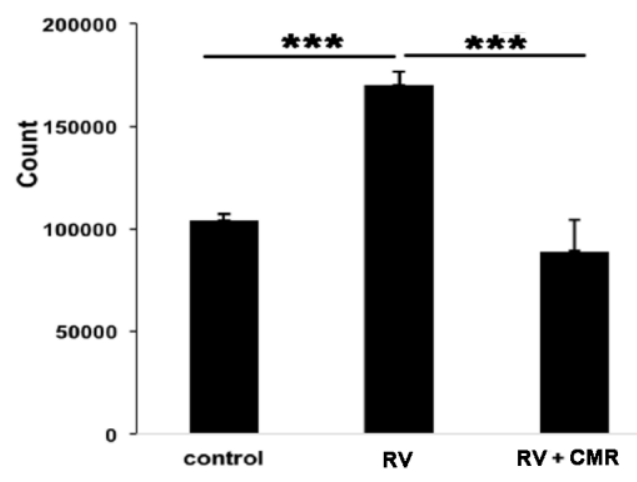

Fig 4. CMR blunts vitreous-induced cell proliferation.

ARPE-19 cells were seeded into a 24 -well plate at a density of $3 \times 10^{4}$ cells/well in DMEM/F12 + 10\% FBS. When attaching the plate, the cells were treated with $0.5 \mathrm{ml}$ DMEM (-) or RV supplemented with CMR (5 $\mu \mathrm{M})$. After treatment for $48 \mathrm{~h}$, the cells were counted with a hemocytometer under a light microscope. The mean \pm SD of the three independent experiments is shown; the data was analyzed using one-way ANOVA followed by the Tukey HSD post hoc-test. *** denotes $\mathrm{p}<0.001$. 
Fig. 5
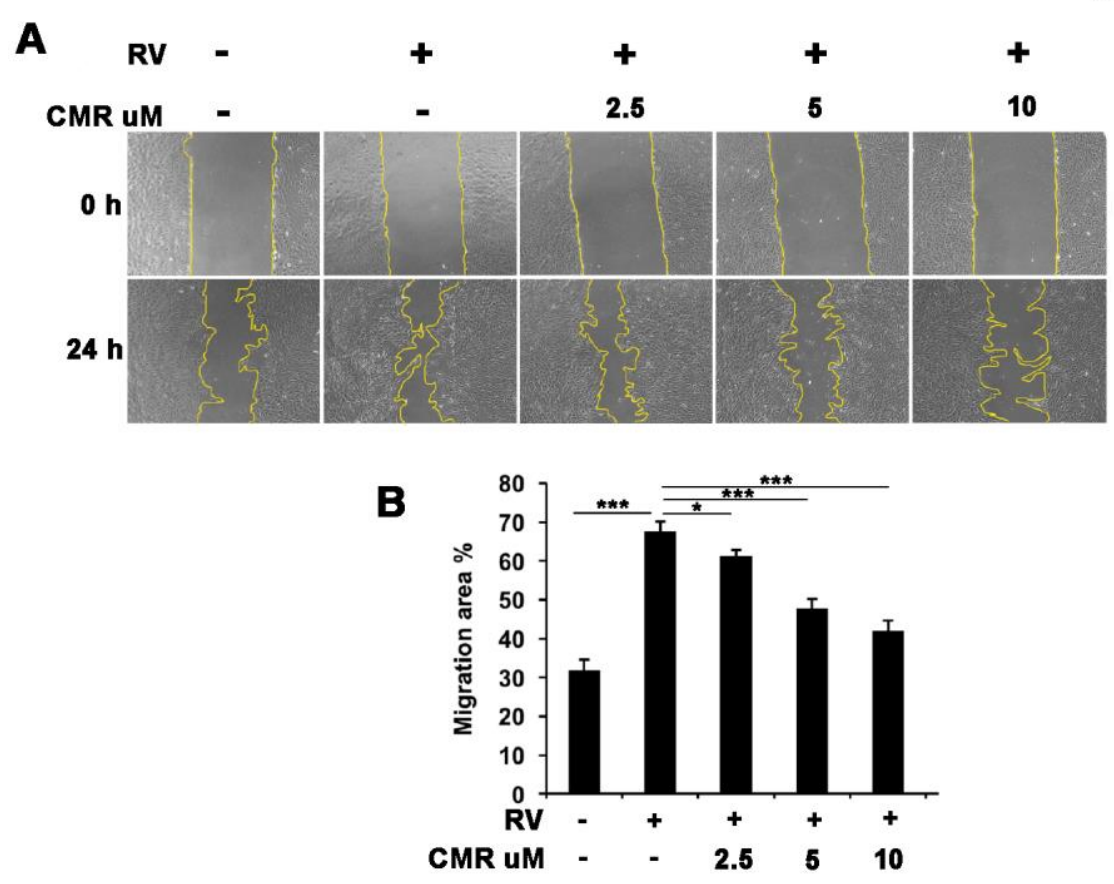

Fig 5. CMR abrogates vitreous-induced cell migration

When ARPE-19 grew to near confluence in a 24-well plate, the wells were scratched using a $200 \mu \mathrm{l}$ pipet tip. The cells were then washed, photographed at an initial width, and treated as described in Fig. 4. 24 h later, images of cells were taken and analyzed for the scratched areas using image $\mathrm{J}$ and Adobe Photoshop CS4 software. The data of bar graphs are the mean \pm SD of three independent experiments. (A) Representative raw data of three independent experiments is shown underneath the bar graphs. (B) * denotes $\mathrm{p}<$ 0.05 , *** denotes $\mathrm{p}<0.001$ using one-way ANOVA followed by the Tukey HSD post hoc-test. 
Fig. 6

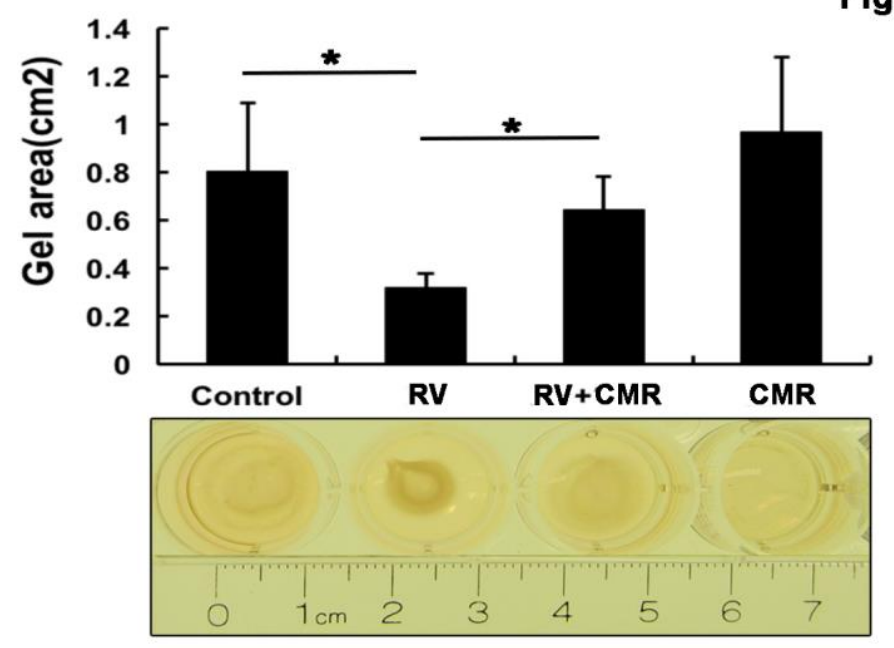

Fig 6. CMR blocks RV-stimulated contraction of ARPE-19.

The ARPE-19 cells were re-suspended in $1.5 \mathrm{mg} / \mathrm{ml}$ of neutralized collagen I (pH 7.2) at a density of $1 \times 10^{6}$ cells $/ \mathrm{ml}$ and seeded into wells of a 24-well plate that had been preincubated overnight with $5 \mathrm{mg} / \mathrm{ml}$ (BSA/PBS). The collagen was solidified by incubation at $37{ }^{\circ} \mathrm{C}$ for $90 \mathrm{~min}$. The polymerized gels were overlaid with DMEM/F12 alone (-) or $\mathrm{RV}$ supplemented with $\mathrm{CMR}(5 \mu \mathrm{M})$ or its vehicle as indicated. $48 \mathrm{~h}$ later, the gel diameter was measured and the gel area calculated using the formula: $3.14 \times$ (diameter/2)2. The bar graphs represent the mean \pm SD of the three independent experiments; *denotes $\mathrm{p}<0.05$ using one-way ANOVA followed by the Tukey HSD post hoc-test. A photograph of the representative experiment in $\mathrm{A}$ is shown at the bottom of the bar graphs. 

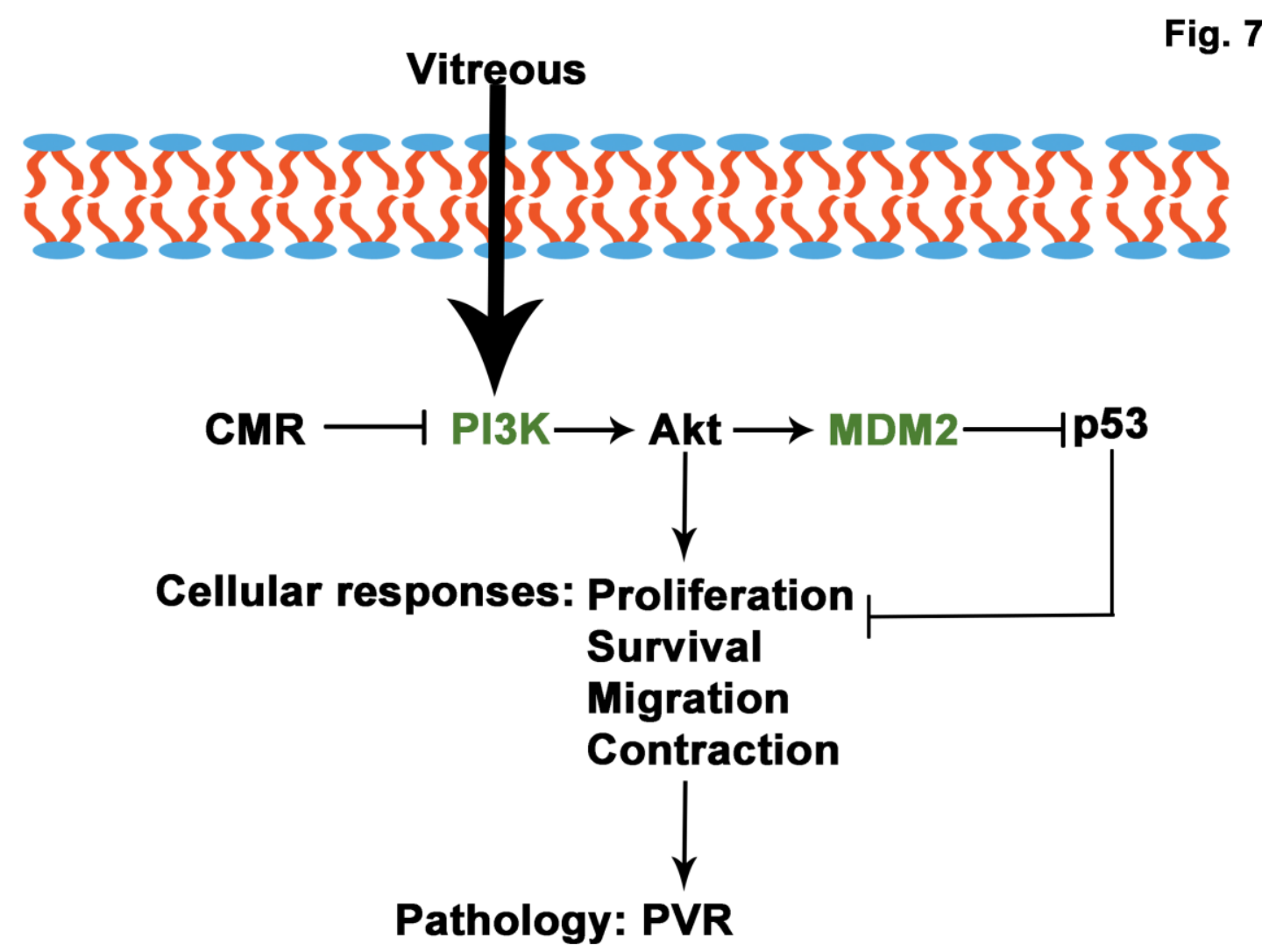

Fig 7. Diagram of CMR inhibiting vitreous-induced activation of Akt/p53 signaling pathway and PVR-related cellular responses including proliferation, migration and contraction. 